01

\title{
Vibrational Spectroscopic and Molecular Docking Studies of Amrinone, a Cardiotonic Inotropic Drug*
}

\author{
(C) Sefa Celik ${ }^{1}$, Sevim Akyuz ${ }^{2}$, Aysen E. Ozel ${ }^{1}$, and Elif Akalin ${ }^{1}$ \\ ${ }^{1}$ Physics Department, Science Faculty, Istanbul University, Vezneciler, \\ 34134 Istanbul, Turkey \\ ${ }^{2}$ Physics Department, Science and Letters Faculty, Istanbul Kultur University, Atakoy Campus, Bakirkoy \\ 34156 Istanbul, Turkey \\ e-mail: scelik@istanbul.edu.tr
}

Received April 29, 2021

Revised April 29, 2021

Accepted September 30, 2021

Amrinone is a class I cardiotonic inotropic agent, which is known to increase the cyclic adenosine monophosphate (cAMP) level by inhibiting the phosphodiesterase 3 (PDE3) enzyme. In this study the theoretically possible stable conformations of the amrinone, was examined first by conformational analysis method and then the obtained most stable conformation was optimized by DFT/wb97xd/6-311++G(d,p) level of theory using Gaussian 03 program. The credibility of the theoretical model was confirmed by comparison of experimental and theoretical vibrational spectra of the title molecule. The fundamental vibrational wavenumbers, IR and Raman intensities of the optimized structure of amrinone were determined using DFT/wb97xd/6-311++G(d,p) level of theory and compared with the experimental vibrational spectra. To investigate the influence of amrinone on cAMP enhancement, the docking simulations towards PDE3B were carried out and the main binding interactions of amrinone with PDE3 were elucidated. Cytochrome P450s (CYPs) are very important phase I metabolizing enzymes. The interaction between amrinone and CYPs (CYP1A2, CYP2C9 and CYP2C19) was investigated by docking simulations. Moreover, molecular docking of the title molecule with different proteins and receptors were studied to reveal potential mechanisms for therapeutic applications. Molecular docking simulations revealed that amrinone showed strong binding affinity to integrins $\alpha_{5} \beta_{1}(\Delta G=-6.6 \mathrm{kcal} / \mathrm{mol})$ and $\alpha_{\mathrm{IIb}} \beta_{3}(-6.6 \mathrm{kcal} / \mathrm{mol})$, and DNA $(-6.5 \mathrm{kcal} / \mathrm{mol})$. The results correlated with its anticancer activity. The drug likeness and ADMET properties of amrinone were analyzed for the prediction of pharmacokinetic profiles.

Key words: amrinone, DFT calculations, FTIR, Molecular Docking, ADMET.

\footnotetext{
* Полный текст статьи опубликован в „Optics and Spectroscopy“ 2022 V. 130. N 02.
} 